Scalable Computing: Practice and Experience, ISSN 1895-1767, http://www.scpe.org (C) 2020 SCPE.

Volume 21, Issues 3, pp. 345-348, DOI 10.12694:/scpe.v21i3.1568

\title{
INTRODUCTION TO THE SPECIAL ISSUE ON EVOLVING IOT AND CYBER-PHYSICAL SYSTEMS: ADVANCEMENTS, APPLICATIONS, AND SOLUTIONS
}

\author{
ANAND NAYYAR, PIJUSH KANTI DUTTA PRAMANIK’ AND RAJNI MOHANA
}

Internet of Things (IoT) is regarded as a next-generation wave of Information Technology (IT) after the widespread emergence of the Internet and mobile communication technologies. IoT supports information exchange and networked interaction of appliances, vehicles and other objects, making sensing and actuation possible in a low-cost and smart manner.

On the other hand, cyber-physical systems (CPS) are described as the engineered systems which are built upon the tight integration of the cyber entities (e.g., computation, communication, and control) and the physical things (natural and man-made systems governed by the laws of physics).

The IoT and CPS are not isolated technologies. Rather it can be said that IoT is the base or enabling technology for CPS and CPS is considered as the grownup development of IoT, completing the IoT notion and vision. Both are merged into closed-loop, providing mechanisms for conceptualizing, and realizing all aspects of the networked composed systems that are monitored and controlled by computing algorithms and are tightly coupled among users and the Internet. That is, the hardware and the software entities are intertwined, and they typically function on different time and location-based scales. In fact, the linking between the cyber and the physical world is enabled by IoT (through sensors and actuators). CPS that includes traditional embedded and control systems are supposed to be transformed by the evolving and innovative methodologies and engineering of IoT.

Several applications areas of IoT and CPS are smart building, smart transport, automated vehicles, smart cities, smart grid, smart manufacturing, smart agriculture, smart healthcare, smart supply chain and logistics, etc. Though CPS and IoT have significant overlaps, they differ in terms of engineering aspects. Engineering IoT systems revolves around the uniquely identifiable and internet-connected devices and embedded systems; whereas engineering CPS requires a strong emphasis on the relationship between computation aspects (complex software) and the physical entities (hardware).

Engineering CPS is challenging because there is no defined and fixed boundary and relationship between the cyber and physical worlds. In CPS, diverse constituent parts are composed and collaborated together to create unified systems with global behaviour. These systems need to be ensured in terms of dependability, safety, security, efficiency, and adherence to real-time constraints. Hence, designing CPS requires knowledge of multidisciplinary areas such as sensing technologies, distributed systems, pervasive and ubiquitous computing, real-time computing, computer networking, control theory, signal processing, embedded systems, etc.

CPS, along with the continuous evolving IoT, has posed several challenges. For example, the enormous amount of data collected from the physical things makes it difficult for Big Data management and analytics that includes data normalization, data aggregation, data mining, pattern extraction and information visualization. Similarly, the future IoT and CPS need standardized abstraction and architecture that will allow modular designing and engineering of IoT and CPS in global and synergetic applications. Another challenging concern of IoT and CPS is the security and reliability of the components and systems.

Although IoT and CPS have attracted the attention of the research communities and several ideas and solutions are proposed, there are still huge possibilities for innovative propositions to make IoT and CPS vision

*Graduate School, Duy Tan University, Da Nang, Vietnam

${ }^{\dagger}$ National Institute of Technology, Durgapur, India

† Jaypee Institute of Information Technology, Wakanghat, India 
successful. The major challenges and research scopes include system design and implementation, computing and communication, system architecture and integration, application-based implementations, fault tolerance, designing efficient algorithms and protocols, availability and reliability, security and privacy, energy-efficiency and sustainability, etc.

It is our great privilege to present Volume 21, Issue 3 of Scalable Computing: Practice and Experience. We had received 30 research papers and out of which 14 papers are selected for publication. The objective of this special issue is to explore and report recent advances and disseminate state-of-the-art research related to IoT, CPS and the enabling and associated technologies. The special issue will present new dimensions of research to researchers and industry professionals with regard to IoT and CPS.

Vivek Kumar Prasad and Madhuri D Bhavsar in the paper titled "Monitoring and Prediction of SLA for IoT based Cloud described the mechanisms for monitoring by using the concept of reinforcement learning and prediction of the cloud resources, which forms the critical parts of cloud expertise in support of controlling and evolution of the IT resources and has been implemented using LSTM. The proper utilization of the resources will generate revenues to the provider and also increases the trust factor of the provider of cloud services. For experimental analysis, four parameters have been used i.e. CPU utilization, disk read/write throughput and memory utilization.

Kasture et al. in the paper titled "Comparative Study of Speaker Recognition Techniques in IoT Devices for Text Independent Negative Recognition" compared the performance of features which are used in state of art speaker recognition models and analyse variants of Mel frequency cepstrum coefficients (MFCC) predominantly used in feature extraction which can be further incorporated and used in various smart devices.

Mahesh Kumar Singh and Om Prakash Rishi in the paper titled "Event Driven Recommendation System for E-Commerce using Knowledge based Collaborative Filtering Technique" proposed a novel system that uses a knowledge base generated from knowledge graph to identify the domain knowledge of users, items, and relationships among these, knowledge graph is a labelled multidimensional directed graph that represents the relationship among the users and the items. The proposed approach uses about 100 percent of users' participation in the form of activities during navigation of the web site. Thus, the system expects under the users' interest that is beneficial for both seller and buyer. The proposed system is compared with baseline methods in area of recommendation system using three parameters: precision, recall and NDGA through online and offline evaluation studies with user data and it is observed that proposed system is better as compared to other baseline systems.

Benbrahim et al. in the paper titled "Deep Convolutional Neural Network with TensorFlow and Keras to Classify Skin Cancer" proposed a novel classification model to classify skin tumours in images using Deep Learning methodology and the proposed system was tested on HAM10000 dataset comprising of 10,015 dermatoscopic images and the results observed that the proposed system is accurate in order of $94.06 \%$ in validation set and $93.93 \%$ in the test set.

Devi B et al. in the paper titled "Deadlock Free Resource Management Technique for IoT-Based Post Disaster Recovery Systems" proposed a new class of techniques that do not perform stringent testing before allocating the resources but still ensure that the system is deadlock-free and the overhead is also minimal. The proposed technique suggests reserving a portion of the resources to ensure no deadlock would occur. The correctness of the technique is proved in the form of theorems. The average turnaround time is approximately $18 \%$ lower for the proposed technique over Banker's algorithm and also an optimal overhead of $\mathrm{O}(\mathrm{m})$.

Deep et al. in the paper titled "Access Management of User and Cyber-Physical Device in DBAAS According to Indian IT Laws Using Blockchain" proposed a novel blockchain solution to track the activities of employees managing cloud. Employee authentication and authorization are managed through the blockchain server. User authentication related data is stored in blockchain. The proposed work assists cloud companies to have better control over their employee's activities, thus help in preventing insider attack on User and Cyber-Physical Devices.

Sumit Kumar and Jaspreet Singh in paper titled "Internet of Vehicles (IoV) over VANETS: Smart and Secure Communication using IoT" highlighted a detailed description of Internet of Vehicles (IoV) with current applications, architectures, communication technologies, routing protocols and different issues. The researchers also elaborated research challenges and trade-off between security and privacy in area of IoV. 
Deore et al. in the paper titled "A New Approach for Navigation and Traffic Signs Indication Using Map Integrated Augmented Reality for Self-Driving Cars" proposed a new approach to supplement the technology used in self-driving cards for perception. The proposed approach uses Augmented Reality to create and augment artificial objects of navigational signs and traffic signals based on vehicles location to reality. This approach help navigate the vehicle even if the road infrastructure does not have very good sign indications and marking. The approach was tested locally by creating a local navigational system and a smartphone based augmented reality app. The approach performed better than the conventional method as the objects were clearer in the frame which made it each for the object detection to detect them.

Bhardwaj et al. in the paper titled "A Framework to Systematically Analyse the Trustworthiness of Nodes for Securing IoV Interactions" performed literature on IoV and Trust and proposed a Hybrid Trust model that seperates the malicious and trusted nodes to secure the interaction of vehicle in IoV. To test the model, simulation was conducted on varied threshold values. And results observed that PDR of trusted node is 0.63 which is higher as compared to PDR of malicious node which is 0.15 . And on the basis of PDR, number of available hops and Trust Dynamics the malicious nodes are identified and discarded.

Saniya Zahoor and Roohie Naaz Mir in the paper titled "A Parallelization Based Data Management Framework for Pervasive IoT Applications" highlighted the recent studies and related information in data management for pervasive IoT applications having limited resources. The paper also proposes a parallelization-based data management framework for resource-constrained pervasive applications of IoT. The comparison of the proposed framework is done with the sequential approach through simulations and empirical data analysis. The results show an improvement in energy, processing, and storage requirements for the processing of data on the IoT device in the proposed framework as compared to the sequential approach.

Patel et al. in the paper titled "Performance Analysis of Video ON-Demand and Live Video Streaming Using Cloud Based Services" presented a review of video analysis over the LVS \& VoDS video application. The researchers compared different messaging brokers which helps to deliver each frame in a distributed pipeline to analyze the impact on two message brokers for video analysis to achieve LVS VoS using AWS elemental services. In addition, the researchers also analysed the Kafka configuration parameter for reliability on full-service-mode.

Saniya Zahoor and Roohie Naaz Mir in the paper titled "Design and Modeling of Resource-Constrained IoT Based Body Area Networks" presented the design and modeling of a resource-constrained BAN System and also discussed the various scenarios of BAN in context of resource constraints. The Researchers also proposed an Advanced Edge Clustering (AEC) approach to manage the resources such as energy, storage, and processing of BAN devices while performing real-time data capture of critical health parameters and detection of abnormal patterns. The comparison of the AEC approach is done with the Stable Election Protocol (SEP) through simulations and empirical data analysis. The results show an improvement in energy, processing time and storage requirements for the processing of data on BAN devices in AEC as compared to SEP.

Neelam Saleem Khan and Mohammad Ahsan Chishti in the paper titled "Security Challenges in Fog and IoT, Blockchain Technology and Cell Tree Solutions: A Review" outlined major authentication issues in IoT, map their existing solutions and further tabulate Fog and IoT security loopholes. Furthermore, this paper presents Blockchain, a decentralized distributed technology as one of the solutions for authentication issues in IoT. In addition, the researchers discussed the strength of Blockchain technology, work done in this field, its adoption in COVID-19 fight and tabulate various challenges in Blockchain technology. The researchers also proposed Cell Tree architecture as another solution to address some of the security issues in IoT, outlined its advantages over Blockchain technology and tabulated some future course to stir some attempts in this area.

Bhadwal et al. in the paper titled "A Machine Translation System from Hindi to Sanskrit Language Using Rule Based Approach" proposed a rule-based machine translation system to bridge the language barrier between Hindi and Sanskrit Language by converting any test in Hindi to Sanskrit. The results are produced in the form of two confusion matrices wherein a total of 50 random sentences and 100 tokens (Hindi words or phrases) were taken for system evaluation. The semantic evaluation of 100 tokens produce an accuracy of $94 \%$ while the pragmatic analysis of 50 sentences produce an accuracy of around $86 \%$. Hence, the proposed system can be used to understand the whole translation process and can further be employed as a tool for learning as well as teaching. Further, this application can be embedded in local communication based assisting Internet of Things (IoT) devices like Alexa or Google Assistant. 
Anshu Kumar Dwivedi and A.K. Sharma in the paper titled "NEEF: A Novel Energy Efficient Fuzzy Logic Based Clustering Protocol for Wireless Sensor Network" proposed a a deterministic novel energy efficient fuzzy logic-based clustering protocol (NEEF) which considers primary and secondary factors in fuzzy logic system while selecting cluster heads. After selection of cluster heads, non-cluster head nodes use fuzzy logic for prudent selection of their cluster head for cluster formation. NEEF is simulated and compared with two recent state of the art protocols, namely SCHFTL and DFCR under two scenarios. Simulation results unveil better performance by balancing the load and improvement in terms of stability period, packets forwarded to the base station, improved average energy and extended lifetime. 